\title{
A Bio-inspired Collision Detector for Small Quadcopter
}

\author{
Jiannan Zhao*, Cheng Hu*, Chun Zhang ${ }^{\dagger}$, Zhihua Wang ${ }^{\dagger}$ and Shigang Yue* \\ ${ }^{*}$ School of Computer Science \\ University of Lincoln,UK Email: \{jzhao, chu, syue\} @lincoln.ac.uk \\ $\dagger$ Department of Microelectronics and Nanoelectronics, Tsinghua University, China \\ Email: \{zhangchun, zhihua\}@tsinghua.edu.cn
}

\begin{abstract}
The sense and avoid capability enables insects to fly versatilely and robustly in dynamic and complex environment. Their biological principles are so practical and efficient that inspired we human imitating them in our flying machines. In this paper, we studied a novel bio-inspired collision detector and its application on a quadcopter. The detector is inspired from Lobula giant movement detector (LGMD) neurons in the locusts, and modeled into an STM32F407 Microcontroller Unit (MCU). Compared to other collision detecting methods applied on quadcopters, we focused on enhancing the collision accuracy in a bio-inspired way that can considerably increase the computing efficiency during an obstacle detecting task even in complex and dynamic environment. We designed the quadcopter's responding operation to imminent collisions and tested this bio-inspired system in an indoor arena. The observed results from the experiments demonstrated that the LGMD collision detector is feasible to work as a vision module for the quadcopter's collision avoidance task.
\end{abstract}

Index Terms-Bio-inspiration, LGMD, Collision avoidance, Locusts vision, Quadcopter

\section{INTRODUCTION}

Quadcopter and its application has become ever more promising, this is because of their ability of agilely flying in real world and exploring extreme environment. Markets pursue flying platform with more intelligence to accomplish robot tasks. Thus, the ability to sense and avoid surroundings is more and more vital for the quadcopter. Traditionally, UAVs use global positioning system (GPS) or optic flow[1][2] to navigate, and use ultra sonic, infrared, laser, or a cooperative system to avoid obstacles as reviewed by [3]. However, it is still challenging for quadcopters to fly automatically in an unfamiliar environment. The SLAM algorithm[4][5] has made progress to address this problem by learning a 3D map of the whole view field, however, it requires too much computing power which constrict this technology to be applied to smaller quadcopters. Thus, we need to study more computing efficient methods for small or micro quadcopters. Nature demonstrates varieties of the successful mechanisms in collision avoidance situation, i.e. the locust is known to have professional fly skills and can fly in millions with out collision. There is a highly specialized neuron in the lobula plate that responds to image luminance, selectively to imminent collision or approaching predators, which is so called: the lobula giant movement detector (LGMD)[6][7]. This neural network has been modeled[7][8] and promoted (e.g. [9][10][11][12]) by

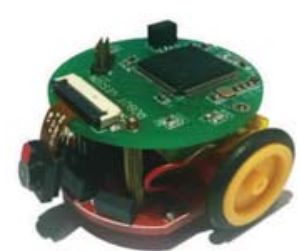

(a) Colias Mobile Robot

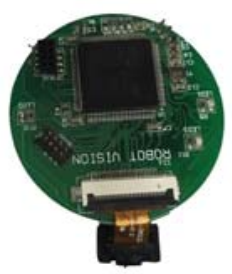

(b) LGMD Vision Detector

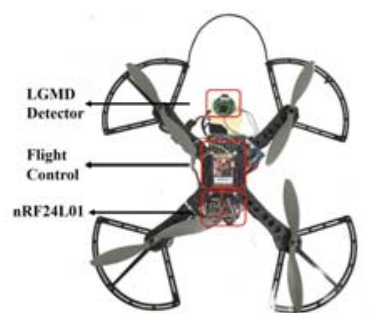

(c) Top view

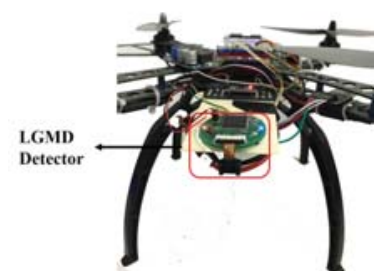

(d) Front view
Fig. 1. Hardware presentation.

previous researchers. The LGMD collision detector has been introduced to mobile robots[13], embedded systems[14][15], cars[16][17], blimp[18], and so on[19]. Whereas, it hasn't been challenged to any faster or more agile aerial vehicles. The LGMD neural network has been introduced to a quadcopter platform[20] by Salt. They novelly put forth an spiking neuron network (SNN) based LGMD model and applied an SNN based vision sensor for vision input. However, few flight experiments have been achieved. Our work is the first time to use the LGMD neural network to achieve a quadcopter's avoiding flight control in real flight, and the results reflected the LGMD detector's features confronting obstacle in a complex environment.

\section{ALGORITHM DESCRIPTION}

The LGMD algorithm used in this paper is inherited from our previous model described in Yue and Rind[10] and Cheng $\mathrm{Hu}[15][13]$ as Fig.2 shows, with some simplification and approximation. The model is composed of five groups of cells, which are P-cells (photoreceptor), I-cells (inhibitory), E-cells (excitatory), S-cells (summing) and G-cells (grouping) and 


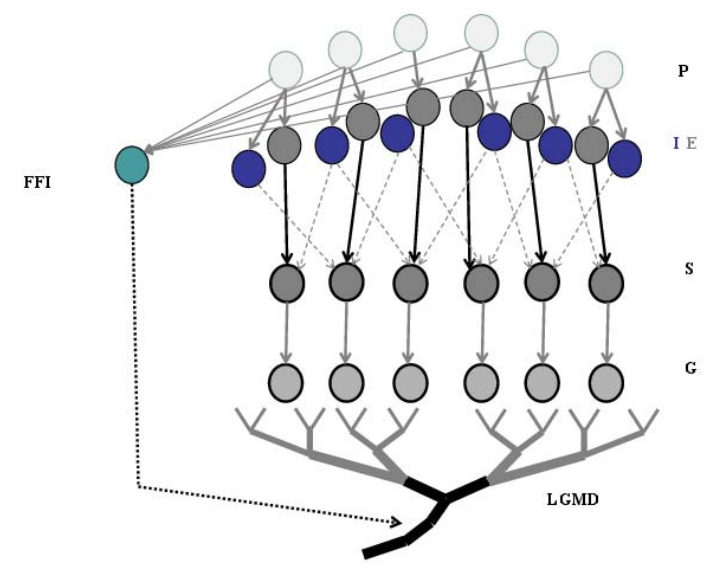

Fig. 2. [10] A schematic illustration of the LGMD based neuron network for collision detection. There are five groups of cells and two single cells. Photorecepter cells (P); Lateral inhibitory and excitatory cells (I and E); Summing cells (S); Grouping cells (G); the LGMD cell and feed forward inhibition cell (FFI); The input of the $\mathrm{P}$ cells is the luminance change, Lateral inhibition is indicated with dotted lines and is delayed by one frame. Excitation is indicated with black lines and without delay. The FFI is also delayed by one frame.

also two individual cells, namely, the feed-forward inhibitory and LGMD.

The first layer of the neuron network is composed of P cells, which are arranged in a matrix, formed by the difference of luminance between adjacent frames which are captured by the camera. Comparing to the define in Yue and Rind[10], a little tail for visual persistence is removed to reduce computation power. The output of a $\mathrm{P}$ cell is given by:

$$
P_{f}(x, y)=L_{f}(x, y)-L_{f-1}(x, y)
$$

where $P_{f}(x, y)$ is the change of luminance of $\operatorname{pixel}(x, y)$ at frame $f, L_{f}(x, y)$ and $L_{f-1}(x, y)$ are the luminance at frame $f$ and the previous frame.

The output of the $\mathrm{P}$ cells forms the input of the next layer and is processed by two different types of cells, which are I (inhibitory) cells and E (excitatory) cells. The E cells pass the excitatory flow directly to $\mathrm{S}$ layer so that the E cells has the same value to its counterpart in P Layer; While the I cells pass the inhibitory flow convoluted by surrounded delayed excitations:

$$
\begin{aligned}
& E_{f}(x, y)=P_{f}(x, y) \\
& \left.I_{f}(x, y)=\sum_{i=-r j=-r}^{r} \sum_{f-1}^{r} P_{f}((x+i),(y+j)) \cdot W(i, j), \quad \text { if } i=j, j \neq 0\right)
\end{aligned}
$$

where the $W(i, j)$ denotes the local inhibition weight matrix and $\mathrm{r}$ indicate the inhibition radius (set to be 2 in our test). In the inhibition layer, It is notable that $\mathrm{i}$ and $\mathrm{j}$ are not allowed to be equal to zero simultaneously. This means the inhibition is only spread out to its neighbouring cells in the next layer rather than to its direct counterpart in the next layer.

The I layer can also be treated as a simplified convolution operation:

$$
[I]_{f}=[P]_{f} \otimes[w]_{I}
$$

where $[w]_{I}$ is the convolution mask representing the local inhibiting weight distribution from the centre cell of $\mathrm{P}$ layer to neighbouring cells in $\mathrm{S}$ layer, a neighbouring cell's local weight is reciprocal to its distance from the centre cell:

$$
[w]_{I}=0.25\left[\begin{array}{ccccc}
\frac{1}{\sqrt{8}} & \frac{1}{\sqrt{5}} & \frac{1}{2} & \frac{1}{\sqrt{5}} & \frac{1}{\sqrt{8}} \\
\frac{1}{\sqrt{5}} & \frac{1}{\sqrt{2}} & 1 & \frac{1}{\sqrt{2}} & \frac{1}{\sqrt{5}} \\
\frac{1}{2} & 1 & 0 & 1 & \frac{1}{2} \\
\frac{1}{\sqrt{5}} & \frac{1}{\sqrt{2}} & 1 & \frac{1}{\sqrt{2}} & \frac{1}{\sqrt{5}} \\
\frac{1}{\sqrt{8}} & \frac{1}{\sqrt{5}} & \frac{1}{2} & \frac{1}{\sqrt{5}} & \frac{1}{\sqrt{8}}
\end{array}\right]
$$

The next layer is the Sum layer, where the excitation and inhibition from the $\mathrm{E}$ and I layer is combined by linear subtraction:

$$
S_{f}(s, y)=E_{f}(x, y)-I_{f}(x, y) \cdot W_{I}
$$

Where $W_{I}$ denotes the inhibition coefficient. However, the excitation would be falsely strengthened by the inhibition flow when using 6 if the inhibition has an opposite sign to the excitation. So an additional condition is significant to constrict the result:

$$
S_{f}(x, y)= \begin{cases}E_{f}(x, y), & \text { if } E_{f}(x, y) \cdot I_{f}(x, y) \leqslant 0 \\ S_{f}(x, y), & \text { otherwise. }\end{cases}
$$

The G layer is introduced to this module in order to reduce the noise from the background. The expanded edges represented by clustered excitations are enhanced to extract colliding objects against complex backgrounds. This layer allows clusters of excitations in the $\mathrm{S}$ cells to easily pass to its corresponding $\mathrm{G}$ cells and provide a greater input to the membrane potential of the LGMD neuron compared with the excitation from a single $\mathrm{S}$ cell. This mechanism is implemented with a passing coefficient for each cell, which is defined by a convolution operation in the $\mathrm{S}$ layer. The passing coefficient is determined by its surrounding pixels, given by:

$$
[C e]_{f}=[S]_{f} \otimes\left[\omega_{e}\right]
$$

where $\omega_{e}$ represents the influence of its neighbours and this operation can be simplified as a convolution mask:

$$
\left[\omega_{e}\right]=\frac{1}{9}\left[\begin{array}{lll}
1 & 1 & 1 \\
1 & 1 & 1 \\
1 & 1 & 1
\end{array}\right]
$$

The excitation correspond to each cell becomes:

$$
G_{f}(x, y)=S_{f}(x, y) C e_{f}(x, y) \omega^{-1}
$$

where $\omega$ is a scale and computed at every frame:

$$
\omega=0.01+\max \left|[C e]_{f} \cdot C_{w}^{-1}\right|
$$


in which $C_{w}$ is a constant and $\max \left|[C e]_{f}\right|$ is the largest absolute value of $C_{e}$. The $\mathrm{G}$ layer is followed by a threshold set to filter decayed excitations:

$$
\widetilde{G}_{f}(x, y)= \begin{cases}G_{f}(x, y), & \text { if } G_{f}(x, y) \geqslant T_{d e} \\ 0, & \text { otherwise. }\end{cases}
$$

Where $T_{d e}$ is the decay threshold. This grouping process can not only enhance the edges of immanent objects, but also filter out the sporadic excitation generated by background details. The membrane potential of the LGMD cell $K_{f}$ is calculated:

$$
K_{f}=\sum_{x} \sum_{y}\left|\widetilde{G}_{f}(x, y)\right|
$$

and then normalized by the equation:

$$
\kappa_{f}=\frac{\tanh \left(\sqrt{K_{f}}-n_{\text {cell }} C_{1}\right)}{n_{\text {cell }} C_{2}}
$$

where $C_{1}$ and $C_{2}$ are constants to shape the normalizing function, limiting the excitation $\kappa_{f}$ varies within $[0,1], n_{\text {cell }}$ represents the total number of pixels in one frame of image.

If the normalised value $\kappa_{f}$ exceeds the threshold, then a spike is produced:

$$
S_{f}^{s p i k e}= \begin{cases}1, & \text { if } \kappa_{f} \geqslant T_{s} \\ 0, & \text { otherwise. }\end{cases}
$$

An impending collision is confirmed if successive spikes last consecutively no less than $n_{s p}$ frames:

$$
C_{f}^{L G M D}= \begin{cases}1, & \text { if } \sum_{f-n_{s p}}^{f} S_{f}^{\text {spike }} \geqslant n_{s p} \\ 0, & \text { otherwise. }\end{cases}
$$

Normally, the LGMD detector generate an "avoid" command if the spike last a few frames $\left(C_{f}^{L G M D}=1\right)$. However, it is not surprised when turning or nodding, a whole-field looming change will leads to false alarm. The feed forward inhibition(FFI) copes with such saccade-like movement by suppress the response to $\left(C_{f}^{L G M D}\right)$. Given that the membrane potential of FFI cell is proportional to the summation of excitations in all cells with one frame delay:

$$
F_{f}=\sum_{x} \sum_{y}\left(\left|P_{f-1}(x, y)\right|\right) n_{c e l l}^{-1}
$$

Once $F_{f}$ exceeds its threshold $T_{F F I}$, spikes in the LGMD are inhibited immediately, the quadcopter will not respond to LGMD spikes in this case:

$$
C_{f}^{F F I}= \begin{cases}1, & \text { if } F_{f} \geqslant T_{F F I} \\ 0, & \text { otherwise. }\end{cases}
$$

In our case, the LGMD result $\left(C_{f}^{L G M D}\right)$ and FFI result $\left(C_{f}^{F F I}\right)$ cooperate to decide the motion state of the quadcopter. The command generated by FFI result has higher priority so that it is able to suppress the response to LGMD in a saccadic-like situation. Motion task switch is handled by a task scheduler explained in Fig.5

The initial values for each parameters are listed in TABLE.1.
TABLE I

INITIAL PARAMETERS OF LGMD BASED NETWORK

\begin{tabular}{c|c|c}
\hline \hline Name & Value & Description \\
\hline$W_{I}$ & 1.0 & $\begin{array}{c}\text { Inhibition Coefficient of inhibition } \\
\text { layer }\end{array}$ \\
\hline$C_{w}$ & 4 & Grouping decaying \\
\hline$T_{F F I}$ & 90 & Threshold of FFI output \\
\hline$T_{d e}$ & 500 & Grouping layer threshold \\
\hline$T_{s}$ & 35 & Spiking threshold for LGMD \\
\hline$n_{c e l l}$ & 7128 & Number of cells \\
\hline$n_{s p}$ & 5 & $\begin{array}{c}\text { Minimal LGMD spike numbers } \\
\text { for a decision }\end{array}$ \\
\hline$C_{1}$ & 150 & Constant for normalization \\
\hline$C_{2}$ & 80 & Constant for normalization \\
\hline \hline
\end{tabular}

\section{SYSTEM OVERVIEW}

In this section, the outline of the whole system is described. To accomplish the obstacle detecting task, luminance information is collected by the camera on the sense board, and then input into the LGMD algorithm, the output is passed through a USART port into the flight control to monitor avoiding tasks.

\section{A. LGMD Vision Detector}

The LGMD vision detector is designed to process image information and to simulate the LGMD neural network on board. It is from the vision module of 'Colias', an open-hardware modular micro robot for swarm robotic applications[21][22]. The detector is mainly consist of a Micro-controller and a CMOS camera. The LGMD algorithm mentioned in the previous part is designed into a 32-bit MCU STM32F407, which clocked at $168 \mathrm{MHz}$ to provides the necessary computational power to have a real-time image processing. It contains 192 Kbyte SRAM that provides enough spaces for image buffing and computing. Images are captured by a CMOS image sensor OV7670 module, which is capable to operating up to 30 frames per seconds(fps) in VGA mode with output support for RGB422, RGB565 and YUV422. The viewing angle is approximately 70 degrees. As a trade-off for image quality and data consumption, we choose a resolution of $72 * 99$ pixels at 30fps, with output format of 8-bit YUV422. The Detector also provides USART interface to transmit results between the flight control module .

\section{B. Quadcopter Platform}

In this paper, we used a DIY quadcopter with the skeleton size of $33 \mathrm{~cm}$ between diagonally rotors as the testbed for the collision detector. The flight control module we used is based on a STM32F407V and provides 5 USART interface for extra peripheral. It is an open source flight control module(http://www.anotc.com) which contains basic posture stabilization algorithm and communication protocol against the ground station, and could be easily modified to accommodate our tasks. A Pix4Flow optic flow module[1] is introduced to generate relative position information and help stabilize the quadcopter. This module usually serve as an alternative of GPS especially in indoor condition where GPS signal is weak or constricted. The source data from the Pix4Flow module is 


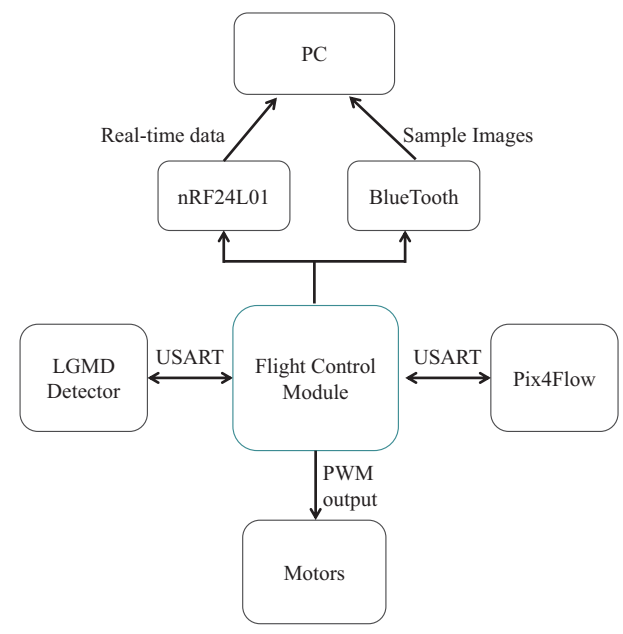

Fig. 3. The structure of the quadcopter platform. The quadcopter is a multi-sensor platform which includes an IMU(Inertial Measurement Unit), an ultrasonic sensor, an optic flow sensor and the LGMD detector. The fligh control module works as the central controller to combine the other parts together. It receives source data from the embedded IMU module(MPU6050), the Pix4flow optic flow sensor, and the LGMD detector, calculates out the PWM(Pulse-Width Modulation) values as the output to the four motors. It also sends back real time data for analysis through the nRF24L01 module and sample images through the bluetooth.

velocity in two axis, this velocity works as the input of a new cascaded PID loop to help nail the quadcopter. In our test, we also integrated the velocity as the approximately position information. The battery is $2200 \mathrm{mAh}$, which can endure 1012 minutes without drop-off.

\section{Ground Station and Supporting Softwares}

Data of the flight control could be transmitted between the flight control module and the off board ground station(PC) through a nRF24L01 $2.4 \mathrm{GHz}$ wireless module. In all the trials, we set the data exchange rate at $100 \mathrm{~Hz}$. In addition, we used a pair of bluetooth module(HC-05) to transmit sample images during trials. One of the bluetooth is connected to the LGMD detector while another is connected to the computer.

\section{Motion Control Mechanism}

In this section, the logical bridge between the output of the LGMD sensor and the UAVs action is elaborated. The mechanism will include how the optic flow sensor is used to estimate the UAVs position and to feedback the control loop.

1) Stabilizing Mechanism: The quadcopter is stabilized by using the algorithm of a cascaded PID loop which is composed of the angular control loop(outer loop) and the angular velocity control loop(inner loop). Traditionally, the input of the outer loop is the data from the remote control, which represents expected angular of the quadcopter. The output of the outer loop is cascaded to the inner loop as the expected angular velocity. The structure of the PID loops is illustrated in Fig.4.

2) Hovering Methods: Generally, the basic cascaded PID control works well to keep the posture of the quadcopter but cannot nail it in the air. That's because we cannot get the

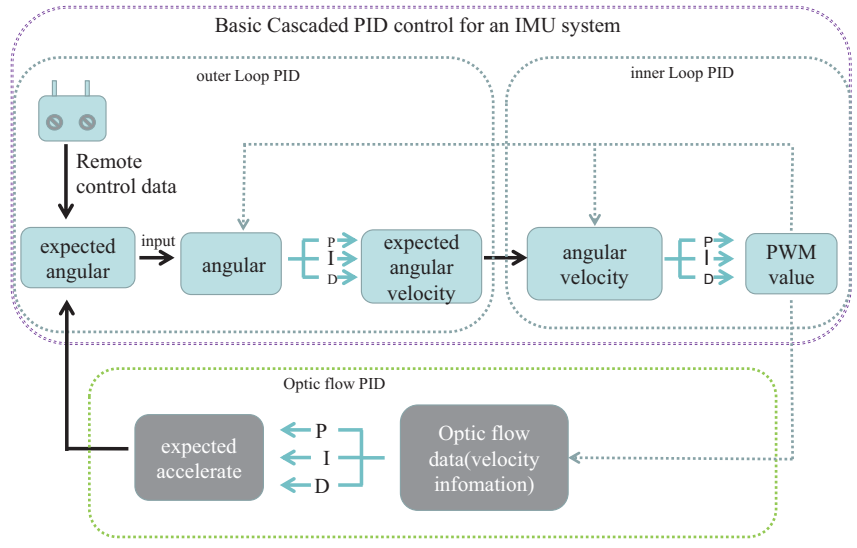

Fig. 4. A schematic of the motion control mechanism. Traditionally, a quadcopter can be stabilized through the basic cascaded PID control loop, and we added the optic flow loop to help the quadcopter to nail itself and to understand its position while executing tasks. Previously, the expected angular is transformed from the Remote control data, and now, it is the algebraic sum of the remote control data and the result of the optic flow PID. The expected accelerate can be transformed to the expected angular because they are relatively proportional while the quadcopter has an insignificant angle of inclination.

accurate velocity of the quadcopter through the accelerometer unless the accumulative error is insignificant. In our test, considering to the indoors condition, to accomplish the hovering function is necessary. Thus, an additional velocity sensor is needed to revise the accumulative error caused by the accelerator. A Pix4Flow sensor is used in our quadcopter. This optic flow sensor supplies the optic flow velocity in two axis, and can be integrated to reflect position information of the quadcopter. We added a new PID control loop for optic flow data, As the velocity information of the quadcopter, the optic flow data is also cascaded to the angular control loop(the outer loop).

3) Task Scheduler: When flying in the arena, the flight is restricted in a 2-D plane. The quadcopter is challenged to switch its motion state in response to impending collision appropriately. The motion state is handled by a task scheduler, which switches tasks among "cruise, avoid and slowdown, depending on the decision made by the cooperation of LGMD and FFI. The quadcopter will fly in straight line if it is clear on the route, and will shift to the side by an approximate distance to avoid the obstacles if the LGMD detected an potential collision. The task flow is illustrated in Fig.5.

\section{EXPERIMENTS AND RESULTS}

As discussed, this bio-inspired collision detector is tested to verify its properties. 3 kinds of tests were implemented to verify the superiority of the LGMD collision detector and its compatibility with quadcopter. 


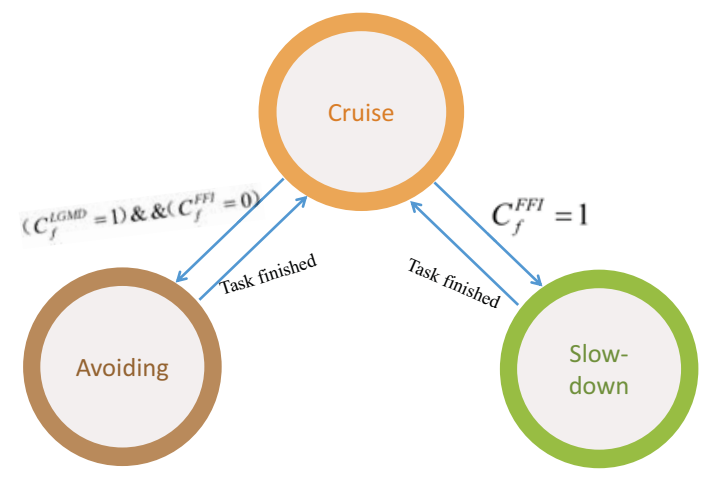

Fig. 5. Task scheduler schematic. The quadcopter works in 'cruise' task in normal situation, which is to fly straightly at an uniform speed, from the start to the destination and vise versa after reach the destination. The task state will change to the other two states in different conditions: a) If the LGMD excitation exceeds the threshold and last $n_{s p}$ frames(which leads to: $C_{f}^{L G M D}=1$ ), the scheduler will invoke the 'avoid' task which process to stop fly ahead and then shift to the lateral side by a defined distance. b) Disparately, once the FFI exceeds the threshold(which leads to: $C_{f}^{F F I}=1$ ), which means huge image motion generated by the quadcopter's own deviation, the interaction to LGMD excitation should be inhibited. In this case, the schedular invokes the 'slowdown' task to weaken the camera's shake.

\section{A. Fixed Detector \& Moving Object Tests}

We first tested the performance of the LGMD Detector confronting factors that cause to luminance change with the detector stationary. Both video simulation and real moving objects have been served as the target and the results are shown in Fig.6 and Fig.7. While confronting the simulated lateral shifting pattern, the responses of the LGMD cells kept almost silent and far from the threshold while the FFI spikes continuously kept at a high value. In this case, when the FFI exceeds its threshold and the LGMD keeps low, a slow-down command will be send to the flight control module because this usually means huge image change is generating by the quadcopter's own deviation. When tested by real moving object, the LGMD is selectively motivated by the approaching jar instead of the lateral shifting one. Compared to traditional frame difference methods, The LGMD neural network is prevented from vulnerably being activated by changing backgrounds. The LGMD collision detector's superiority in differentiating complex backgrounds and approaching foregrounds comes from the lateral inhibition neural structure, the experiments demonstrated that the LGMD neural network is an ideal model in differentiating changing backgrounds and approaching targets.

\section{B. Features analysis \& Parameters adjustment}

Before the quadcopter is pushed to accomplish avoiding tasks, we analyzed the features and characters of the LGMD when the quadcopter is flying in the Arena. The detector's parameters are adjusted to the degree that it hardly alarms falsely towards backgrounds despite surrounding obstacles. The quadcopter is also tested to ensure that it responds differently when image motion is generated by itself deviation and

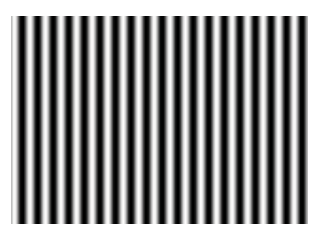

(a) A sample frame of the simulated video

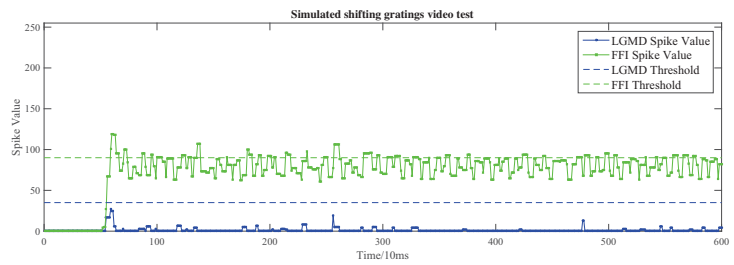

(b) Spiking results in gratings simulation

Fig. 6. Neural responses for video simulation. A simulated lateral shifting gratings pattern is presented in front of the detector's camera, the result is sent back through the nRF24101 module on the quadcopter at the frequency of $100 \mathrm{~Hz}$ (the same as the following: Fig.7(c),Fig.7(d), Fig.8(b), Fig.10(b)). The responses of the LGMD cells kept almost silent and far from the threshold while the FFI spikes continuously kept at a high value. This result explains the utility of the lateral inhibition layer, which is to suppress the response to lateral shifting things and slowly changing backgrounds so that the LGMD neural network only interests to quickly moving/approaching object.

by approaching object, see Fig.8. The experiment is carried out while the quadcopter is hovering in the arena, an external force is given to the quadcopter to make it rotates. The huge image change exceeds the ability of the lateral inhibition cells, so both the FFI and LGMD spikes exceeds their threshold successively. We adjusted the threshold of FFI to make it easily reached when face to such huge image change, so that the FFI spikes increase so quickly to hit the line that the LGMD spikes cannot stay sufficient frames upon the threshold. In this case, The response of the LGMD is ignored and a slow down command is generated to reduce influences from self-rotation once the FFI hit the threshold line.

\section{Obstacle Avoiding Test}

Finally, we tested this bio-inspired method with the quadcopter to challenge its performance in obstacle avoiding case. The arena is indoors, as shown in Fig.9, flex banner with special texture is put on the ground to enhance the accuracy of the optic flow sensor. A box(pasted with textured paper) is set in the middle of the room, as the 'obstacle'. Our task is to let the quadcopter flies automatically approaching the obstacle and avoid it automatically by the command generated from the LGMD detector. In the experiments, we have carried out 6 trials to collect the data. In each trial, the quadcopter starts from flying towards the obstacle. Once it detected the imminent collision, it shifted left to avoid the collision. The trajectories of these trials have been extracted and overlaid on a screenshot of the scene, as shown in Fig.11. Trajectories are detected by a python program using background subtractor[23] and template matching[24] method, and then printed onto a 

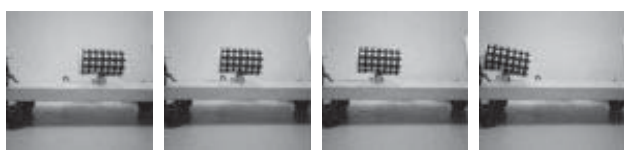

(a) Sample images for lateral shifting
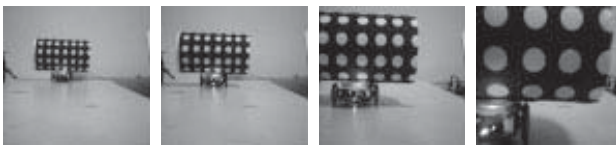

(b) Sample images for approaching object

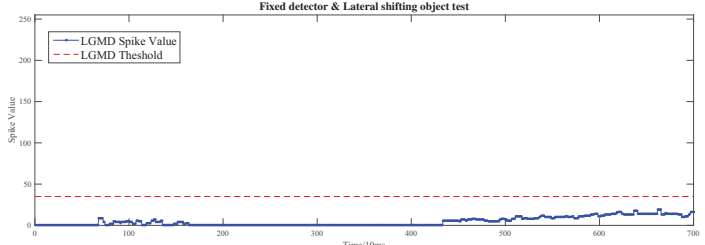

(c) Result of laterally shifting objects test.

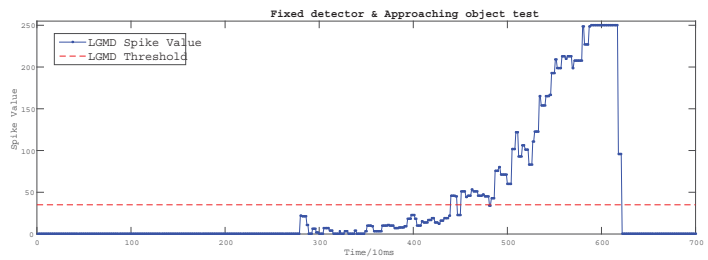

(d) Result of approaching objects test.

Fig. 7. Spiking results for moving object with the detector fixed. We put a jar onto a small mobile robot(Colias) and let the robot moves laterally or towards the camera from the same start point. As the result shows, the spike value keeps at a low level when face to lateral shifting object, while increases quickly towards approaching object. In the approaching object test, the imminent collision is detected at around $460(10 \mathrm{~ms})$, and the spike value increases continuously until the jar hit the camera at around $600(10 \mathrm{~ms})$

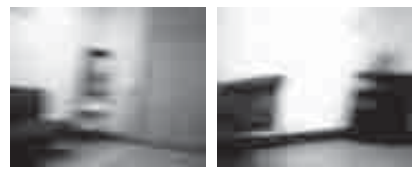

(a) Sample frames of the surrounding during self-rotation

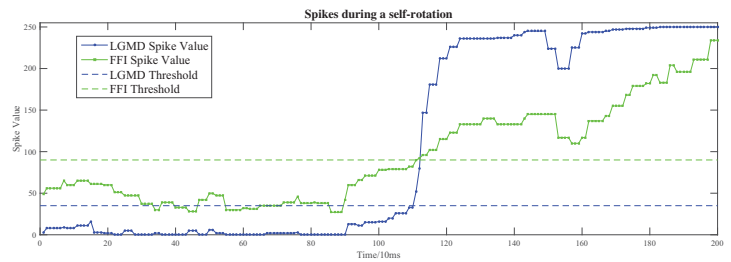

(b) Spiking results caused by self-rotation

Fig. 8. Self-rotation test. In this case, We give an external force to the quadcopter, and make the quadcopter rotates while its hovering in the air. The both line exceeds their threshold but the FFI spikes increase so quickly to hit the line that the LGMD spikes cannot stay sufficient frames upon the threshold. Thus, the response of the LGMD is ignored and a slow down command is generated to reduce influences from self-rotation.

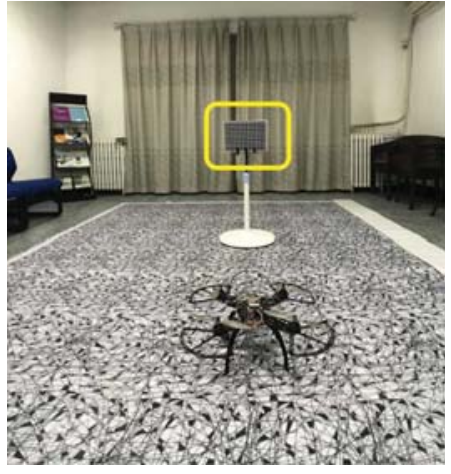

Fig. 9. A glimpse of the arena. Obstacle highlighted with yellow rectangle
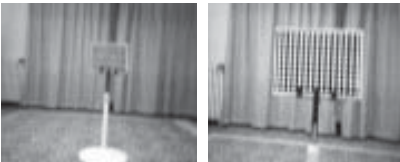

(a) Sample frames when heading the obstacle

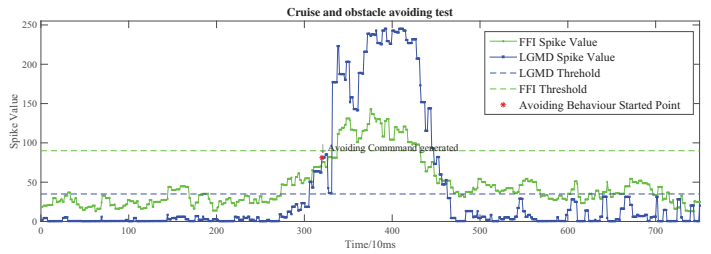

(b) Spiking results in avoiding test

Fig. 10. Result of the obstacle avoiding test in the arena. The green line is the output of the FFI layer; The blue line is the output of the G layer(LGMD cell); The obstacle was first detected at the point marked with red star, and the quadcopter succeeded to avoid the obstacle before colliding. The excitation keeps a high level during the avoiding process, where we close the response to the repeated excitation until an avoiding process finished.

screen shot from the overlook camera. In all the experiments, the quadcopter had successfully avoided the obstacle with the LGMD detector. A typical spiking result of the network during an avoiding test is shown in Fig.10(b). Both FFI and LGMD spike value increase while approaching the obstacle, the LGMD spike climbs quickly and stayed enough frames over the threshold. Thus, at the point marked with red star, the LGMD detector generated an avoiding command and alarmed the flight control module to execute the avoiding movement.

\section{CONCLUSION}

In the above sections, the bio-inspired vision detector is challenged on a quadcopter platform to accomplish obstacle avoiding task. The results shows the reliability and efficiency of this novel method. The approaching selectivity (compared to lateral shifting objects and changing backgrounds) and computing efficiency are the main priority of this bio-inspired method. The LGMD collision detector is capable to cope with coming collisions for a quadcopter platform, and has the potential to cooperate with the other current exiting collision avoidance solutions. 


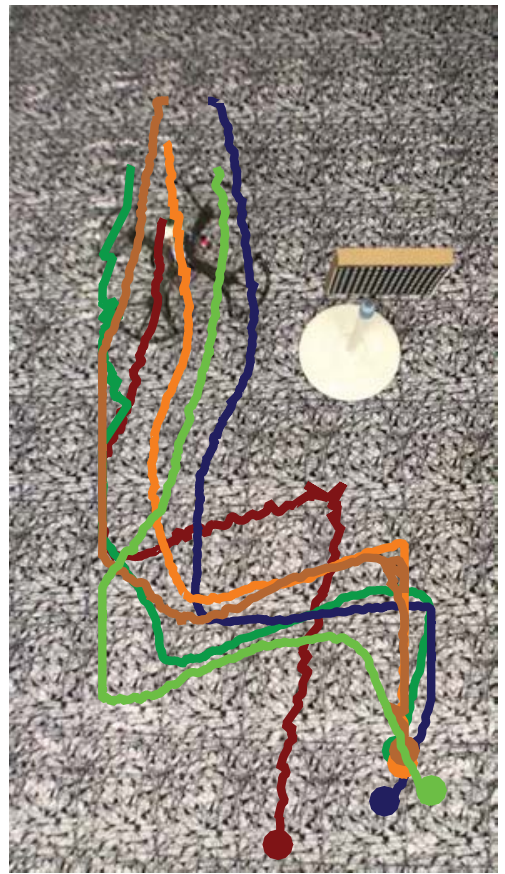

Fig. 11. Automatically flying trajectories during the obstacle avoidance test. Several trajectories of the center point of the quadcopter were printed onto the image with different color, the starting point is decorated by dots. The trajectories show clearly that the quadcopter can avoid the obstacle appropriately before striking it.

\section{ACKNOWLEDGMENT}

The authors would like to thank Xuelong Sun and Qingbing $\mathrm{Fu}$ for their discussion during the research period. And this work was supported by EU FP7 project LIVECODE (295151), HAZCEPT (318907), HORIZON 2020 project STEP2DYNA (691154).

\section{REFERENCES}

[1] D. Honegger, L. Meier, P. Tanskanen, and M. Pollefeys, "An open source and open hardware embedded metric optical flow cmos camera for indoor and outdoor applications," in Robotics and Automation (ICRA), 2013 IEEE International Conference on. IEEE, 2013, pp. 1736-1741.

[2] C. Sabo, A. Cope, K. Gurny, E. Vasilaki, and J. Marshall, "Bio-inspired visual navigation for a quadcopter using optic flow," AIAA Infotech@ Aerospace, vol. 404, 2016.

[3] X. Yu and Y. Zhang, "Sense and avoid technologies with applications to unmanned aircraft systems: Review and prospects," Progress in Aerospace Sciences, vol. 74, pp. 152-166, 2015

[4] A. J. Davison, I. D. Reid, N. D. Molton, and O. Stasse, "Monoslam: Real-time single camera slam," IEEE transactions on pattern analysi and machine intelligence, vol. 29, no. 6, pp. 1052-1067, 2007.

[6] F. C. Rind, "A chemical synapse between two motion detecting neurones in the locust brain." Journal of Experimental Biology, vol. 110, no. 1, pp. $143-167,1984$.
[5] B. Steder, G. Grisetti, C. Stachniss, and W. Burgard, "Visual slam for flying vehicles," IEEE Transactions on Robotics, vol. 24, no. 5, pp. 1088-1093, 2008.

[7] — "Motion detectors in the locust visual system: from biology to robot sensors," Microscopy research and technique, vol. 56, no. 4, pp 256-269, 2002.

[8] R. D. Santer, R. Stafford, and F. C. Rind, "Retinally-generated saccadic suppression of a locust looming-detector neuron: investigations using a robot locust," Journal of The Royal Society Interface, vol. 1, no. 1, pp. 61-77, 2004

[9] S. Yue, R. D. Santer, Y. Yamawaki, and F. C. Rind, "Reactive direction control for a mobile robot: a locust-like control of escape direction emerges when a bilateral pair of model locust visual neurons are integrated," Autonomous Robots, vol. 28, no. 2, pp. 151-167, 2010.

[10] S. Yue and F. C. Rind, "Collision detection in complex dynamic scenes using an lgmd-based visual neural network with feature enhancement," IEEE transactions on neural networks, vol. 17, no. 3, pp. 705-716, 2006.

[11] Q. Fu and S. Yue, "Modelling lgmd2 visual neuron system," in Machine Learning for Signal Processing (MLSP), 2015 IEEE 25th International Workshop on. IEEE, 2015, pp. 1-6.

[12] Q. Fu, C. Hu, T. Liu, and S. Yue, "Collision selective lgmds neuron models research benefits from a vision-based autonomous micro robot," in Intelligent Robots and Systems (IROS), 2017 IEEE/RSJ International Conference on. IEEE, 2017, pp. 3996-4002.

[13] C. Hu, F. Arvin, C. Xiong, and S. Yue, "A bio-inspired embedded vision system for autonomous micro-robots: the lgmd case," IEEE Transactions on Cognitive and Developmental Systems, vol. PP, no. 99, pp. 1-1, 2016.

[14] Q. Fu, S. Yue, and C. Hu, "Fu, yue, hu: Bio-inspired collision detector with enhanced selectivity for ground robotic vision system," in $B M V C$, 2016.

[15] C. Hu, F. Arvin, and S. Yue, "Development of a bio-inspired vision system for mobile micro-robots," in Joint IEEE International Conferences on Development and Learning and Epigenetic Robotics. IEEE, 2014, pp. 81-86.

[16] S. Yue, F. C. Rind, M. S. Keil, J. Cuadri, and R. Stafford, "A bio-inspired visual collision detection mechanism for cars: Optimisation of a model of a locust neuron to a novel environment," Neurocomputing, vol. 69, no. 13 , pp. 1591-1598, 2006

[17] S. Yue and F. C. Rind, "A synthetic vision system using directionally selective motion detectors to recognize collision," Artificial life, vol. 13 , no. 2, pp. 93-122, 2007.

[18] S. Bermudez i Badia, P. Pyk, and P. F. Verschure, "A fly-locus based neuronal control system applied to an unmanned aerial vehicle: the invertebrate neuronal principles for course stabilization, altitude control and collision avoidance," The International Journal of Robotics Research, vol. 26, no. 7, pp. 759-772, 2007.

[19] S. B. i Badia, U. Bernardet, and P. F. Verschure, "Non-linear neurona responses as an emergent property of afferent networks: A case study of the locust lobula giant movement detector," PLoS computational biology, vol. 6, no. 3, p. e1000701, 2010.

[20] L. Salt, G. Indiveri, and Y. Sandamirskaya, "Obstacle avoidance with lgmd neuron: towards a neuromorphic uav implementation," in Circuits and Systems (ISCAS), 2017 IEEE International Symposium on. IEEE, 2017, pp. 1-4.

[21] F. Arvin, J. Murray, C. Zhang, and S. Yue, "Colias: An autonomous micro robot for swarm robotic applications," International Journal of Advanced Robotic Systems, vol. 11, no. 7, p. 113, 2014.

[22] F. Arvin, A. E. Turgut, T. Krajník, and S. Yue, "Investigation of cuebased aggregation in static and dynamic environments with a mobile robot swarm," Adaptive Behavior, vol. 24, no. 2, pp. 102-118, 2016.

[23] Z. Zivkovic, "Improved adaptive gaussian mixture model for background subtraction," in Pattern Recognition, 2004. ICPR 2004. Proceedings of the 17th International Conference on, vol. 2. IEEE, 2004, pp. 28-31.

[24] J. P. Lewis, "Fast normalized cross-correlation," in Vision interface, vol. 10 , no. 1,1995 , pp. 120-123. 